\title{
Outcomes of the Nutri-Senex Project: A Co-Ordination Action Funded by the European Commission
}

\author{
I. Elmadfa ${ }^{a}$ H. Freisling ${ }^{a} \quad$ M. Kueck ${ }^{b} \quad$ I. Matullat $^{b} \quad$ G. Hall ${ }^{c} \quad$ A. Azpiroz ${ }^{d}$ \\ M. Kiely ${ }^{e}$ for the Nutri-Senex Consortium \\ ${ }^{a}$ Department of Nutritional Sciences, University of Vienna, Vienna, Austria; ${ }^{b}$ ttz Bremerhaven, Bremerhaven, \\ Germany; ' SIK - The Swedish Institute for Food and Biotechnology, Gothenburg, Sweden; ${ }^{d}$ SENDATEK,

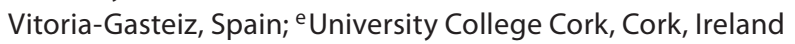

\section{Key Words}

Quality of life $\cdot$ Malnutrition · Elderly $\cdot$ Healthy food

options $\cdot$ Research coordination

\begin{abstract}
This project's primary aim is to contribute to the improvement of the quality of life of the elderly. The state of the art in age-related research and technology, to highlight the most promising areas for development, has been documented in an extensive literature report. A research database has been established to map current research activities within the European research area. Through several expert group meetings with representatives from universities, research institutions, organizations concerned with the care of the elderly, and industry, suitable technologies and policies that can improve the diet of the elderly have been identified. A legislation survey relating to aspects of nutrition of the elderly has been carried out. A set of guidelines and recommendations of best practice for use in the health and care industry have been developed. The project's results are disseminated through an enhanced website, technology transfer workshops and a staff training program. Good commu-
\end{abstract}

nication between the partners has been promoted and permanent research links have been forged. The main outputs will lead to better co-ordinated European research: less duplication of effort, more rapid commercialization, increased awareness of the problems faced by the elderly, improved guidelines and recommendations for health care professionals and policymakers.

Copyright $\odot 2008$ S. Karger AG, Basel

\section{Introduction}

The aging of the world's population is the result of the continued decline in birth rates and increased life expectancy. The economically more developed regions of the world have been most affected by population aging since the beginning of the 20th Century; however, it is an increasing problem in other areas of the world. Older adults, often the frail elderly referred to in this project, comprise the fastest growing portion of the world's population.

A large area of research into malnutrition of the elderly is already being covered. However, the objectives are largely research based and the project participants are

\section{KARGER}

Fax +41613061234 E-Mail karger@karger.ch www.karger.com (c) 2008 S. Karger AG, Basel

0250-6807/08/0525-0072\$24.50/0

Accessible online at:

www.karger.com/anm
Prof. I. Elmadfa

University of Vienna, Department of Nutritional Sciences

Althanstrasse 14

AT-1090 Wien (Austria)

Tel. +43 14277549 11, Fax +43 1427795 49, E-Mail ibrahim.elmadfa@univie.ac.at 
comprised mostly of academic organizations. This project has been sought to co-ordinate the efforts and results of this research, and implement strategies to disseminate the key findings. The project has not only brought together institutions active in the area of food research and technology, but also health care professionals, government/national bodies involved with the elderly, food manufacturers, NGOs, and charities representing senior citizens. The overall objective was to increase the standard of living for the frail elderly in the medium to long term by helping to develop healthier foods and by spreading best practice.

The project's primary aim was to contribute to the improvement of the quality of life of the elderly. Its benefits are likely to be felt most keenly by the frail elderly, those that require constant care by others, but its activities are not confined to this subgroup. The global objectives were to co-ordinate research into the nutrition of the elderly, to improve their quality of life, to reduce public health costs through the prevention of nutrition-elated diseases, and to encourage the development of nutritionally balanced food products specially designed for the elderly. By this, the project aimed to increase understanding of the dietary requirements of this vulnerable group, and how their nutrition can assist or hinder their healthy aging. This should encourage the development and bringing to market of food products that are specifically designed to cater for the altered sensory perception of the aging population, principally the institutionalized elderly for whom food is selected by professional carers.

The specific objectives of this co-ordination action were to:

- Co-ordinate European research activities in nutrition for the elderly

- Document the state of the art in age-related research and technology, and to highlight the most promising areas for development

- Map current research activities within the ERA

- Identify suitable technologies and policies that can improve the diet of the elderly, through studies and expert group meetings

- Survey legislation relating to care for and nutrition of the elderly, highlighting loopholes and best practice

- Improve the understanding of healthy food options available to care providers for the elderly

- Develop a set of guidelines and recommendations of best practice for use in the health and care industry

- Assist the implementation of these guidelines through two implementation workshops in the first and second years of the project
- Disseminate the project's results through an enhanced website, technology transfer workshops and a staff training program

- Promote good communication between the partners and forge permanent research links

The project had ambitious goals and, in order to meet them best, the workplan was structured into seven dedicated work packages (WPs), which represent the main areas of activity in the network.

WP1 was concerned with project co-ordination and further development, and was responsible for all of the other work packages. WP1 includes the resources for all project meetings, which were held biannually after the kick-off meeting, and the steering committee meetings that were held alongside them. WP1 was also responsible for developing the project's web site, keeping the partners informed of the project's progress and their obligations, and for satisfying the EC's reporting requirements.

\section{Mapping of Current Research Activities and Centers of Excellence (WP2)}

WP2 assembled much of the fundamental knowledge that underpinned the project's other work packages. A state-of the-art review was conducted using literature and patent searches performed by members of the project. This allowed the partners to assess the current state of the art, and to identify which technologies are suitable for further exploitation in WP6 (implementation strategies). Further literature and patent searches on an annual basis ensured that the project's members remain abreast of the latest developments in the field. Another key strand of WP2 was a mapping of centers of excellence in research into nutrition and care for the elderly, which can be used to identify the leading players in the European Research Area in preparation for an expansion of the consortium and future projects in that area of research. The main milestone from this section of the workplan was the first input of information into the database of European research activities. This, and an analysis of its effectiveness by questionnaire, was used to gauge its ease-of-use and improve it. Identification of the leading players in the European Research Area was the other main milestone. 


\section{Co-Ordination of European Research Activities (WP3)}

One aim of the Nutri-Senex project was to bring European researchers and experts from universities, food institutes, food industry and organizations together to discuss and recommend research within the area of aging, food, nutrition, and health. The activities within WP3 were divided into two main tasks: (1) the organizing of research co-ordination seminars, and (2) the organizing of expert group discussions.

The purpose of the research co-ordination seminars was to get a state-of-the-art description of on-going European research within some areas of high relevance to healthy aging. Three public seminars were organized, with contributions from Nutri-Senex partners as well as from invited speakers. Among the topics discussed at these seminars were: aging and nutrition; aging, chemoreception, and changes in sensory capabilities; aging, life style, and food choice. Outcomes of other EU-funded projects (HealthSense and Food in Later Life) were also presented.

The purpose of the expert group discussions was mainly to identify research needs in some areas of high relevance to healthy aging. The selected themes, which reflected the special expertise of those who participated in Nutri-Senex, were aging and nutrition (needs, risks, and recommendations), aging, sensory capability, and food perception; healthy aging in relation to lifestyle, and food choice. The groups met for discussion on three occasions. The final outcome was a summarizing recommendations report based on the conclusions drawn from the discussions. It was clearly recognized that this is an area for multidisciplinary research ranging from individual nutrients to consumer behavior, and that much research is still missing. The European dimension, with all its cultural differences, was emphasized. This presentation will give a brief summary of the recommendations.

\section{Analysis of Legislations and Recommendations for the Future (WP4)}

WP4, which run in parallel with WP2 and WP3, was another important component of the project. It built a complete picture of existing legislation at European, national and local levels regarding food provision, looking in particular at any controlling legislation or recommended standards specifically for the care industry. The effect of current legislation on the provision of food in institutions, care homes, etc. was assessed, and strategies that such institutions have adopted to apply such legislative developments were highlighted. Beneficial effects of this legislation were reported, as were potential drawbacks or areas where improvements could be made. A report of recommendations and guidelines for future standards and legislation to promote the use of suitable food products for the frail elderly across Europe was the main goal of this WP. This report was presented at a special meeting to interested stakeholders (January, 2006, Amsterdam, The Netherlands) and widely disseminated in digital and paper form. The key milestone was the completion of the legislation review, which was improved based upon feedback received at the legislation workshop analyzed (June 2006, Leatherhead, UK), prior to its final circulation to interested stakeholders.

\section{Mapping of Product Development (WP5)}

WP5 is a short, yet important, part of the project. Its first task was to construct a database of interested food producers and manufacturers, research institutions, and nongovernmental agencies involved in food, nutritional, or gerontological research. Activities aimed at promoting the uptake of healthier food for the frail and institutionalized elderly were studied and their effectiveness gauged. By applying these findings, and results from other parts of the Nutri-Senex project, a short report was produced detailing how best to encourage the uptake of foods designed to promote long-life and well-being amongst this vulnerable, and ever more numerous, sector of society. The main milestone was the identification of industrially relevant research and technological development (RTD) findings. Key updates to the database at the end of years 2 and 3 of the project, and resulting analyses of any change, were the work packages' other two milestone points.

Most of the findings from WP5, and the earlier work packages, were applied actively by WP6 (Implementation Strategies). During this work package, key organizations working within the health care sector and health and nutrition professionals had an opportunity to meet with researchers and food manufacturers to discuss findings and consider how best to achieve a real impact on the health of older citizens. This was done primarily through implementation workshops, where key problems were discussed and possible solutions outlined. These workshops lead to the development of a guidance booklet, advising health care professionals, nursing staff, etc. of the 
specific dietary needs of elderly citizens, and how best these could be met. Analysis of the effectiveness of both strategy workshops, based on questionnaires circulated to participants, was the main milestones in this work package.

\section{Implementation Strategies (WP6)}

In order to decide which strategies to employ in the short, medium, and long term, implementation strategies aimed at forming contacts with key stakeholders involved in policymaking and strategy workshops were used. The results were then applied to balance the needs of elderly people and food providers with the interests of the general public and of food manufacturers.

Based on this, key organizations have been invited to attend the first annual strategy workshop celebrated in Lyon in 2005 and the second implementation strategy workshop in 2006 at Leatherhead Food International. The main conclusions from the first workshop are:

- Information and assessment should be directed to the care staff, health professionals, as well as cooks and the elderly

- Edition and proper dissemination of a practical guide, as well as training in its proper understanding and use

- Governments and institutions should be made aware of their role mainly in relation to infrastructures and health lifestyle promotion

- However, relation of satisfaction to well-being is considered a poor research level.

The importance of economic issues in the implementation strategies should also be considered. Main conclusions from the second workshop:

- 'Elderly' is not a homogeneous population group, but a condition, and many different groups with different needs might be found, so one single strategy is not valid; multidisciplinary teams are needed to get results

- Need of political and economical support for promotion of healthy ways of life

- Importance of nutritional attention for the entire lifecycle.

Taking into account the conclusions from both implementation workshops, a booklet containing recommendations directed to workers of care homes (managers, nurses and care-staff and cooks) as well as to elderly and their relatives and friends has been prepared.

In order to facilitate the reading of these recommendations, the booklet was formatted in 4 different fact sheets, one for each of the above-mentioned occupational groups. Project volunteers have translated these documents into different languages so that it might be presented in the mother tongue of the readers in at least 10 European countries.

\section{Dissemination and Technology Transfer (WP7)}

The aim of WP7 was the dissemination of the project results and the technology transfer. These objectives were carried out by establishment of a web site, organizing technology transfer workshops, and organizing staff secondments. Additionally, the general project promotion was realized by promotional leaflets, posters, and presentations distributed to the main stakeholders at conferences and trade fairs.

The goal of the web site was to be a powerful dissemination tool and an interface between the network and the external community interested in nutrition and aging. This involved setting up advanced communication links (a topic-specific newsgroup, chat rooms, web conferencing facilities, etc.). The technology transfer workshops, coordinated by TTZ, were prepared and run by experts in the field. The main topic for the Technology Transfer Workshops was 'Trends and needs of an aging population: influence on an aging process towards a healthier society'.

The staff secondment program involved the training of personnel, whereby Consortium Members were able to benefit from others' experience and technology practices adopted at other organizations. The staff secondment proved to be a very good training tool for the partners. The best ideas and discussions are usually born and developed at such meetings and work shops. Questions can be asked to better understand the work of the respective partner and thus the 'visitor' has the opportunity to gain an insight into the partner's field of work.

\section{Conclusion}

By this European Co-ordination Action Project, the consortium believes that the following outputs were achieved:

- Better co-ordinated European research: less duplication of effort, more rapid commercialization

- Increased awareness of the problems faced by the elderly 
- Improved guidelines and recommendations for health care professionals and policymakers

- Improved quality of life for the elderly, through an improvement in the range and quality of food products aimed at this sector of society.

\section{Further Reading}

www.univie.ac.at/nutri-senex

\section{Disclosure Statement}

A Co-ordination Action Funded by the European Commission under the Food Quality and Safety Priority Thematic Area. Contract Number: Food-CT-2003-506382. 